\title{
D-STAR MAC Protocol: a Cross Layer Solution for Wireless Sensor Networks Endowed with Directive Antennas
}

\author{
Gianfranco Manes, Romano Fantacci, \\ Francesco Chiti, Michele Ciabatti, Giovanni Collodi, Davide Di Palma, Ilaria Nelli, and Antonio Manes. \\ Department of Electronics and Telecommunications - University of Florence, \\ via di S. Marta 3, I-50139 Firenze, Italy. \\ Email: name.surname@unifi.it
}

\begin{abstract}
This paper deal with a novel MAC layer protocol, namely Directive Synchronous Transmission Asynchronous Reception (D-STAR) able to logically synchronize a Wireless Sensor Network (WSN) by managing both sleep/active states together with integrating directional antennas within the communications framework, according to a cross-layer design. D-STAR protocol has been characterized in terms of functional characteristics and the overall performance is presented, in terms of network lifetime gain, set-up latency and collision probability. It is shown a remarkable gain with respect to the basic approach endowed with omnidirectional antennas without increasing the signaling overhead nor affecting the set up latency, but achieving energy consumption reduction.
\end{abstract}

Index Terms-Wireless Sensor Networks, directive antennas, MAC protocols design, energy efficiency, performance evaluation.

\section{INTRODUCTION}

Wireless sensor networks (WSNs) [1] have been attracting a great deal of scientific interest in the last decade, making of this approach an enabling technology to the aim of intelligent environments instrumenting. The deployment of networks comprised of tens up to thousands of sensors currently represents an affordable solution to some challenging problems: environmental sensing, productive chains control, real-time phenomena monitoring, safety and rescue application.

Though WSNs represent a special case of the more general wireless Ad Hoc networks paradigm [2], they differs from it for additional constraints, as far as the limited energetic, storage, processing and communication capabilities, the low degree of mobility and the presence of a small number of sinks. However, recent advances have lead to the emergence of distributed wireless sensor and actor networks (WSANs) [3] which are capable of enhanced capabilities of observing, data processing and making. This represents a more critical scenario as the usual impairments of ad hoc communications must be taken also into account.

This innovative paradigm basically relies on a underlying paradox claiming at pursuing reliable tasks through intrinsically unreliable actors. This challenge might be got over

This work was supported in part by the EU Integrated Project FP6-IST1-508774-IP "GoodFood" as well as by the EU Network of Excellence FP6-IST-4-027738-NoE "CRUISE" and EU STREP FP6-IST-045299-STREP "DustBot". through careful system design with particular regard to the communications and control protocols. It is of particular relevance whenever advanced interaction and sensing schemes are applied as it happens in the case of WSANs or mobile WSNs.

Among the most promising criteria to be adopted, the proper management of both sleep state and active power and the introduction of directional antennas and their integration within the communications framework play a key role [4]. As these aspects belong to both the Physical (PHY) and Medium Access Control (MAC) layers, they might be integrated to reach an overall energy efficiency, jointly managing the duty cycle $\delta$ and the transmitting and receiving antenna gains, $G_{t}$ and $G_{r}$, respectively. A novel and promising approach to accomplish this task relies and the so called cross-layer protocol design principle [5]. On the other hand, the increased system complexity needs to be addressed and possibly limited as well as the capability of quickly set up an end-to-end communication path.

This paper aims at filling this gap by proposing a novel MAC layer protocol, namely Directive Synchronous Transmission Asynchronous Reception (D-STAR), that broaden the previously introduced STAR approach [6] toward the management of directive antennas neither increasing the signaling overhead nor affecting the set up latency, but achieving energy consumption reduction and remarkable lowering of collision probability. To this end, the cross-layer principle has been adopted to design a protocol that adapts physical parameters (as the antenna main lobe) to the link-to-link communications features and, on the other hand, provides a nodes' logical synchronization explicitly taking into account the antenna capabilities.

The paper is organized as follows. In Section II, the proposed D-STAR MAC protocol features are properly described. To this end, a general framework to deal with effective MAC protocols for WSNs present in the literature is preliminary introduced (Sec. II-A). Finally, Sec. II-B deals with the proposed approach, giving a deep insight in terms of functional characteristics, finite state machine (FSM) description and the related protocol time charts for different use cases. The overall communications protocol performance is presented, in terms of network lifetime gain, set-up latency and collision probability, 
in Section III. Finally, some conclusions are drawn explaining the future directions of the present research activity.

\section{PRoposed MAC PROTOCOL}

\section{A. Related works}

WSNs differ from wireless ad hoc networks because of more energy constraints: nodes employed in WSNs are characterized by limited resources such as storage, processing and communication capabilities [1], [4]. While the use of smart antennas in ad hoc networks has been proposed [2], their application in the WSN field is highly desirable for several reasons: higher antenna gain might compensate the reduced coverage range due to higher frequencies (for realizing small size nodes) or to preserve connectivity in networks and efficiently use the node energy thus increasing its lifecycle. Besides, the adoption of smart antennas allows the gain maximization toward the desired directions by concentrating the energy in a smaller area, with a transmitted power decreasing, a received power increasing, a power consumption reduction, a network lifetime increasing, an error probability reduction and a coverage range increasing, with a better network capacity. Moreover, as the joint application of smart antennas and channel access management protocol permits to reduce the power radiation toward undesired, it is possible reducing interference caused by other transmissions and collision probability.

To achieve these benefits, there has been a lot of interest in the design of new protocols; however, the use of these antennas presents significant challenges at the higher layers of the protocol stack; in particular, the medium access and the routing layers will have to be modified. In the following, a brief overview of the protocol design solutions in the presence of directional antennas as proposed in the literature, is examined.

Several extensions of IEEE 802.11 protocol have been proposed. The basic protocol, named Directional MAC (DMAC) [7], operates in two phases: the first one (based on directional RTS/CTS exchanges) is used for tuning the receiver antenna on the sender direction and after this phase, DATA and ACK messages are exchanged directionally. Overhearing nodes update their directional network allocation vectors as in IEEE 802.11. This protocol suffers from several problems due to the directional nature of the communications and the increased directional range: hidden terminals due to unheard RTS/CTS messages, hidden terminals to asymmetry in gain and deafness.

Multi-hop MAC (MMAC) [8] extends the basic DMAC protocol by using multi-hop RTSs to establish a directional link between sender and receiver. Then CTS, DATA and ACK are transmitted over a single hop by exploiting the directional antennas gain. The advantage of doing this is to reduce the hop-counts on routes and in bridging possible network partitions. The basic problems with hidden terminals and deafness still exist with the MMAC protocol; however, the benefits due to the use of the increased range somewhat compensates for the other negative effects.

The Receiver-Oriented Multiple Access (ROMA) [9] protocol has been proposed for scheduled access with the presence of multi-beam antenna arrays (MBBA). It exploits the multilink feature of MBBA so a node can commence several simultaneous communication sessions by forming up to $K$ links, where $K$ indicates the number of antenna beams. Besides, the system is capable of performing omnidirectional transmissions and receptions.

\section{B. Directive STAR MAC Protocol}

The proposed Directive STAR (D-STAR) MAC protocol expands the STAR MAC concept [6] allowing a time-space synchronization. The network infrastructure is built up by means of bidirectional links joining: each node sends to its neighbors its own phase, while the angular position is implicitly taken into account at the transmitter and receiver sides, so that nodes are able to communicate. It is worth noticing that this approach is mainly suited for flat networks in which there are no cluster heads distributing a time frame and for densely deployed networks with a number of neighbors per node greater than ten [6].

To attain this goal D-STAR provides a functional scheme comprised of three main phases. To give an exhaustive description of the protocol it is possible to refer to the state diagram given in Fig. 1.

According to it, every node wakes up independently from the other ones and enters an initial idle mode (INIT), in which it remains for a time interval necessary to perform the elementary CPU operations. Then it switches into the DISCOVERY phase where it tries to recognize its neighbors and to establish a logical synchronization with them. Within this phase the operation mode of $j^{\text {th }}$ node is duty cycled with a listening and sleeping sub-periods, whose duration is $T_{l, j}$ and $T_{s, j}$, respectively, periodically repeated ${ }^{1}$.

For the sake of generality, it has been supposed that the generic $j^{\text {th }}$ node has its own frame period $T_{f, j}$ and duty cycle $\delta_{j}$ (and of course listening $T_{l, i}$ and sleeping $T_{s, i}$ subperiods) with $j=1, \ldots, N$ and $N$ the total number of network nodes. Moreover, it has been assumed that the wake up time is randomly selected by each node.

To provide an affordable, less complex and robust solution, during the initial set-up phase each node remains in a listening mode for a time interval equal to:

$$
T_{\text {set-up }} \geq 2 \max _{j}\left\{T_{f, j}\right\}
$$

During the discovery phase each node begins to broadcast one HELLO messages to each angular sector (i.e., the coverage area within a certain side lobe) sending its $I D$ and phase; then it waits for a fixed time duration $\tau_{s}$ in search of reply messages $^{2}$ and switches to the following angular sector, repeating the procedure until $T_{\text {set-up }}$ is expired.

In particular, each node sends the HELLO messages with a period:

$$
T_{\text {broad }} \leq \min _{j}\left\{T_{l, j}\right\}
$$

\footnotetext{
${ }^{1}$ The abrupt introduction of this operation mode allows a remarkable power saving as it is avoided a unnecessary long listening phase, while more attention might be devoted to minimize also the set-up latency.

${ }^{2}$ Once the communication is logically established with this node, the following HELLO messages sent to it a a unicast way are able to reach also the other nodes within the same angular sector.
} 
As a consequence, the number of HELLO messages sent by the $j^{\text {th }}$ node during the discovery phase is equal to:

$$
N_{\text {broad }} \geq \frac{T_{f, j}}{\min _{i}\left\{T_{l, i}\right\}} N_{s}
$$

where $N_{s}$ is the number of non-overlapping angular sectors of the transmitter smart antenna ${ }^{3}$. The value of the phase $\phi$ sent is strictly related to the time interval remaining to exit the discovery phase and enter the duty cycled mode.

It is worth noticing that as $N_{s}$ increases the cost of HELLO messages transmission is predominant with respect to the cost of the listening mode for the vast majority of hardware platform available on the market. This might justify a posteriori the simplified exit condition from the set-up phase.

Once the discovery phase is expired, each node enters the regime phase, according to Fig. 1. The reference node then sends HELLO messages in unicast way to the neighbors belonging to different angular sectors according to the phase transmitted in previous HELLO messages, but also sends several HELLO messages in background with the proper period to unknown neighbors in the empty angular sector. Upon the replying of a node, a logical channel is established and $j^{\text {th }}$ node can adopt the unicast or the multicast approach, according to the STAR+ approach [6]. Again, the transmitted phase value $\phi$ is the time interval after which the sender claims to be again in the listening status.

The channel access is managed by means of a CSMA/CA (Carrier Sense Multiple Access with Collision Avoidance) approach. Before transmitting a packet toward a certain angular sector, a node first listens to the channel: if no transmitted packets are detected, it assumes that the channel is clear and starts transmitting. Otherwise, it must wait and try again the transmission in that sector after a random time interval until a maximum number of attempts has been reached. This mechanism is very effective in reducing collisions, while the problem of hidden node [2] is still partially unsolved.

Each node remains in the regime phase until there is at least one neighbor, otherwise if the number of empty angular sector is equal to $N_{s}$ it re-enters the discovery phase in search of connectivity ${ }^{4}$.

Whenever a node battery is depleted, this node turns off, entering an $O F F$ phase both from the discovery phase and from regime phase. This transition is not represented in Fig. 1.

\section{PERformance AnAlysis}

To evaluate the performance of the proposed D-STAR protocol, extensive numerical simulations has been conducted over a realistic scenario in compliance with the pilot site developed by EU Integrated Project "GoodFood" [10]. The most relevant simulation parameters are summarized in Table I. The adopted antenna model is an ideal switched beam antenna. It has been supposed to create a group of almost non-overlapping beams that together result in omnidirectional coverage, so that the patterns' main lobes are adjacent. The microcontroller at each

\footnotetext{
${ }^{3}$ The value of $\max _{i}\left\{T_{f, i}\right\}$ might be estimated by each node on the basis of its own characteristics and the same is true for $\min _{i}\left\{T_{l, i}\right\}$.

${ }^{4}$ It might be due to the fact that a node could have joined the network extremely late or even have changed its position.
}

node is able to scan the channel according to the D-STAR protocol, switching to the correct beam corresponding with the user wishing to communicate at that time. Only a single beam pattern is employed at any given time. In particular, the antenna has been conceived such that to cover a fixed arc or sector of, say, $\pi, \pi / 2, \pi / 3$ and $\pi / 4$ radiants, thus providing increased gain over a restricted range of azimuths as compared to an omnidirectional antenna. Besides, WSN nodes are supposed to be deployed only in a 2D scenario.

The proposed approach has been conceived mainly to minimize the power consumption, thus enhancing the network lifetime ${ }^{5}$. To this end, a duty cycled operation and directive antennas have been introduced and properly managed to allow full connectivity thorough time-space synchronization. However, D-STAR protocol is also able to minimize the setup latency, as the discovery phase duration $T_{\text {set-up }}$ is upper bounded by twice the maximum frame period value, as explained in (1).

To give an insight on the protocol energy efficiency, in Fig. 2 the lifetime as a function of number of network nodes has been pointed out in the case of omnidirectional antennas (that is the basic STAR MAC protocol), and directive antennas with two or four angular sectors, respectively. It could be noticed the remarkable gain provided by the introduction of directive antennas; in particular, it is almost equal to 4 or 16 in the case of two or four angular sectors, respectively, in accordance with analytical predictions. Nevertheless, performance gets worst as the number of nodes increases due to the presence of packets collisions that implies packets retransmissions and transmitted power wasting. It is not surprisingly that the network lifetime is extremely high ${ }^{6}$ as it has been simulated only the MAC layer operations without taking into account the packet forwarding at the Network layer that is undoubtedly the most relevant cause of power consumption.

In Fig. 3, the same comparisons have been performed with respect to the duty cycle value which has been varied over a commonly adopted range of $[1 \%, 5 \%]$. Without pointing out again the noticeable gain, it is possible to highlight that lifetime keeps contact no matter what the dutycycle is, as the larger the listening time the greater the receiving cost and the lower the collision probability.

Finally, in Fig. 4, the network lifetime as a function of the frame period duration $T_{f}$ is shown. Within a usual operation range for $T_{f}$ from $10 \mathrm{~s}$ up to $90 \mathrm{~s}$, the lifetime has a linear increase as listening sub-period $T_{l}$ is also proportional to $T_{f}$ and it mostly effects the overall power consumption.

The energy efficiency of the proposed D-STAR protocol can be evaluated also focusing on the collision probability that depends upon the node density and the presence of the hidden nodes. The underlying CSMA/CA mechanism might fail indeed if neighbor nodes get extremely close or if two or more nodes not belonging to the same coverage area attempt to transmit toward the same node.

To get an insight on this aspect, in Fig. 5, the collision probability as a function of the number of network nodes is

\footnotetext{
${ }^{5}$ As to our purpose, the network lifetime has been assumed in a strict sense, i.e., as the time interval after which the first node is turned off.

${ }^{6}$ For instance it is equal to two years and half in the worst case.
} 
depicted, again in the case of omnidirectional antennas and directive antennas with two, four, six, eight possible angular sectors, respectively. It could be noticed that the adoption of omnidirectional antennas minimize the packets collisions even in the case of densely deployed nodes, while the converse is true for directive antennas mostly due to the presence of hidden nodes, as the coverage area gets smaller in terms of azimuth and an increasing number of nodes become invisible ${ }^{7}$. However, as the angular resolution increases, a lower number of nodes might interfere with a third node when transmitting and the communications become really point-to-point. This effect is more evident in the case of directive antennas with a number of possible angular sector greater than four as for lower values a sort of spatial blindness is always present ${ }^{8}$.

The adoption of a medium access scheme following a CSMA/CA approach implies that a new channel sensing is randomly scheduled whenever a channel is not detected as idle. This allows to avoid packet collision, whilst reducing the link throughput. To conclude this analysis, Fig. 6 points out the probability of finding the channel occupied as a function of the number of deployed nodes. In this case the most conservative scheme, that is the omnidirectional one, highlights the worst behavior for this parameters being compensated by a better collision probability, while the opposite happens for more directional antennas.

\section{CONClusions And Further Developments}

WSN paradigm it is widely considered as the most promising for intelligent environments instrumenting, leading to novel communications scenarios. However, this could be pursued by means of effective protocols design, since WSN nodes present specific constraints, as far as the limited resources, the low degree of mobility and the unattended operations.

This paper deal with both the sleep/active states power management as well as the introduction of directional antennas and their integration within the communications framework, following a cross-layer design. A novel MAC layer protocol, namely Directive Synchronous Transmission Asynchronous Reception (D-STAR) is proposed, aiming at expanding the capabilities of previously introduced STAR MAC approach [6] toward the management of directive antennas neither increasing the signaling overhead nor affecting the set up latency, but achieving energy consumption reduction.

D-STAR protocol has been characterized in terms of functional characteristics, state transitions diagram representation and the related time charts for different use cases. The overall communications protocol performance is presented, in terms of network lifetime gain, set-up latency and collision probability, pointing out a remarkable gain with respect to the basic approach endowed with omnidirectional antennas.

Future developments of the present research activity might include the protocol implementation and testing over a a realistic user defined scenarios like those proposed in [10],

\footnotetext{
${ }^{7}$ It could be noticed that in any case the maximum value for collision probability keeps lower that $2 \%$.

${ }^{8}$ This statement is true in the case of a symmetric link, i.e., with the same antenna at the receiver and transmitting sides. Otherwise, the performance are limited by the antenna with lower directivity.
}

\begin{tabular}{|c|c|}
\hline Parameter & Value \\
\hline \hline Monitored Area $\left[\mathrm{m}^{2}\right]$ & $25 \times 25$ \\
\hline Number of nodes & {$[10, \ldots, 50]$} \\
\hline Number of angular sectors & {$[1,2,4,6,8]$} \\
\hline Frame duration $T_{f}[\mathrm{~s}]$ & {$[10,25,50,75,93]$} \\
\hline Dutycycle $\delta[\%]$ & 0 \\
\hline Transmitted power [dBm] & -50 \\
\hline Receiver attenuation [dBm] & -90 \\
\hline Receiver sensitivity [dBm] & {$[1, \ldots, 5,1,2,3,4]$} \\
\hline Transmitting antenna gain $G_{t}$ & {$[0.5,1,2,3,4]$} \\
\hline Receiving antenna gain $G_{r}$ & 2500 \\
\hline Battery initial level [mAh] & $6 \cdot 10^{-5}$ \\
\hline Cost of 1 HELLO pkt transmission [mAh] & $2.777 \cdot 10^{-3}$ \\
\hline Cost of 1 HELLO pkt reception/channel sensing [mA/s] & $2.97 \cdot 10^{-6}$ \\
\hline Cost of sleeping [mA/s] & 6 \\
\hline Number of channel sensing with CSMA/CA & 0.02 \\
\hline Time duration of a channel sensing attempt $[\mathrm{s}]$ & 8 \\
\hline Hello packet size [B] & 250 \\
\hline Transmission Bit-rate [kb/s] & 86400 \\
\hline Packet error-rate [\%] & \\
\hline Simulated time interval [s] & 5 \\
\hline
\end{tabular}

TABLE I

PARAMETERS VALUES ADOPTED WITHIN THE NUMERICAL SIMULATION CAMPAIGN.

[11] as well as the application to fields different from the environmental monitoring as the emergency operations with specific requirements [12].

\section{REFERENCES}

[1] I. F. Akyildiz, W. Su, Y. Sankarasubramaniam, and E. Cayirci, "Wireless Sensor Networks: a survey," IEEE Computer Networks, vol. 38, pp. 393422, March 2002.

[2] P. Mohapatra and S. V. Krishnamurthy, Ad Hoc Networks-Technologies and Protocols. Springer Science \& Business Media Inc., 2005.

[3] I. F. Akyildiz and I. H. Kasimoglu, "Wireless Sensor and Actor Networks: research challenges," Elsevier Ad Hoc Networks Journal, vol. 2, no. 4, pp. 351-367, October 2004.

[4] A. Hać, Wireless Sensor Networks Designs. John Wiley \& Sons, 2003.

[5] S. Shakkottai, T. S. Rappaport, and P. C. Karlsson, "Cross-layer Design for Wireless Networks," IEEE Communications Magazine, vol. 41, pp. 74-80, October 2003.

[6] F. Chiti, M. Ciabatti, G. Collodi, D. D. Palma, R. Fantacci, and A. Manes, "Design and Application of Enhanced Communication Protocols for Wireless Sensor Networks operating in Environmental Monitoring," in Proc. of IEEE ICC'06, 2006.

[7] C. Perkins, Ad Hoc Networks. Addison-Wesley, 2000.

[8] L. Breslau, D. Estrin, K. Fall, S. Floyd, J. Heidemann, A. Helmy, P. Huang, S. McCanne, K. Varadhan, Y. Xu, and H. Yu, "Adavance in Network Simulation," IEEE Computer, vol. 33, no. 5, pp. 59-67, MAY 2000.

[9] L.Bao and J. Garcia-Luna-Aceves, "Transmission Scheduling in Ad Hoc Networks with Directional Antennas,' in Proc. of MOBICOM'02, Sept. 2002.

[10] "EU Integrated Project FP6-IST-1-508774-IP "GoodFood"." [Online]. Available: http://www.goodfood-project.org.

[11] "EU Network of Excellence FP6-IST-4-027738-NoE "CRUISE"." [Online]. Available: http://www.ist-cruise.eu.

[12] “EU STREP FP6-IST-045299-STREP "DustBot"." [Online]. Available: http://www.dustbot-project.org. 


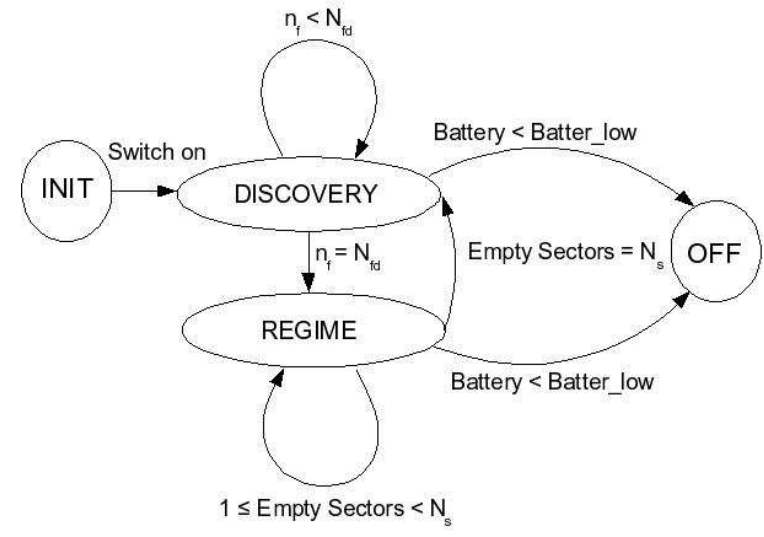

Fig. 1. Finite state machine description of the proposed D-STAR protocols with the related phases.

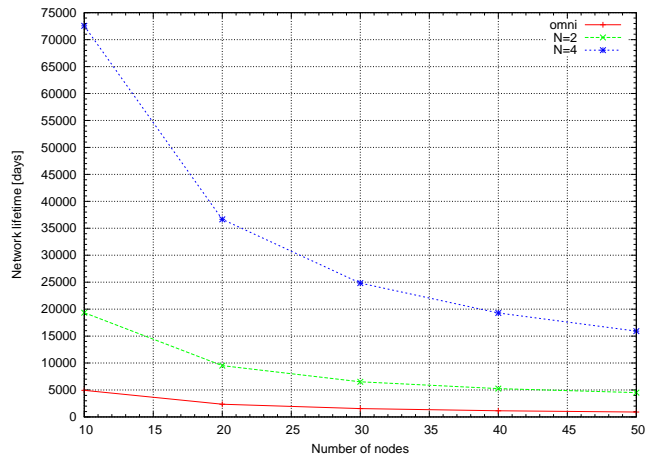

Fig. 2. Network lifetime as a function of the number of nodes in the case of omnidirectional and directive antennas with $\pi$ or $\pi / 2$ mainlobes for $T_{f}=$ $93 s$ and $\delta=3 \%$.

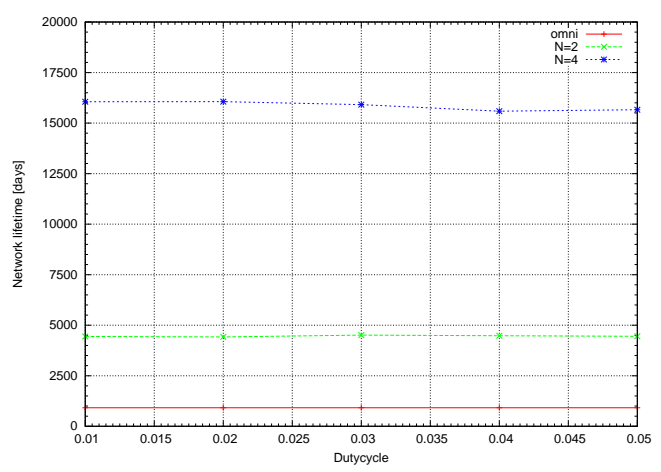

Fig. 3. Network lifetime as a function of the dutycyle $\delta$ in the case of omnidirectional and directive antennas with $\pi$ or $\pi / 2$ mainlobes for $T_{f}=$ $93 s$ and 50 nodes.

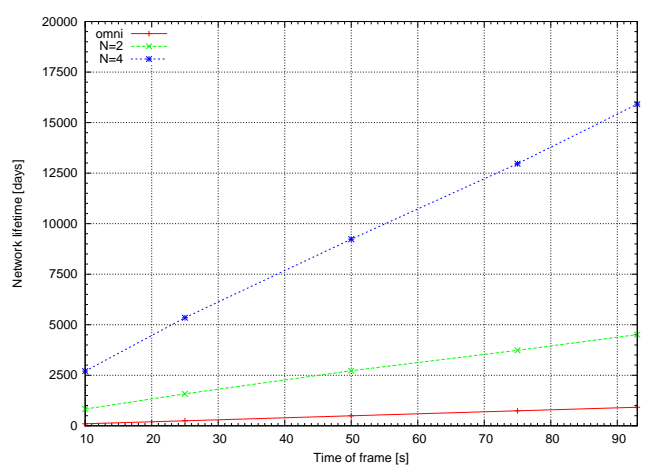

Fig. 4. Network lifetime as a function of the frame duration $T_{f}$ in the case of omnidirectional and directive antennas with $\pi$ or $\pi / 2$ mainlobes for $T_{f}=93 s$ and 50 nodes.

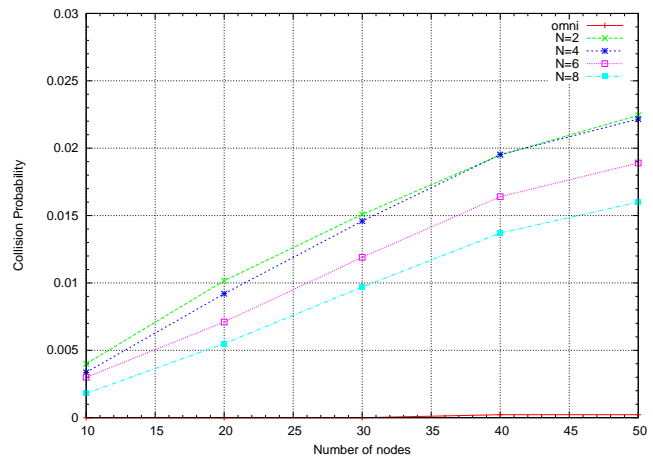

Fig. 5. Collision probability as a function of the number of nodes in the case of omnidirectional and directive antennas with $\pi, \pi / 2, \pi / 3$ and $\pi / 4$ mainlobes for $T_{f}=93 s$ and $\delta=3 \%$.

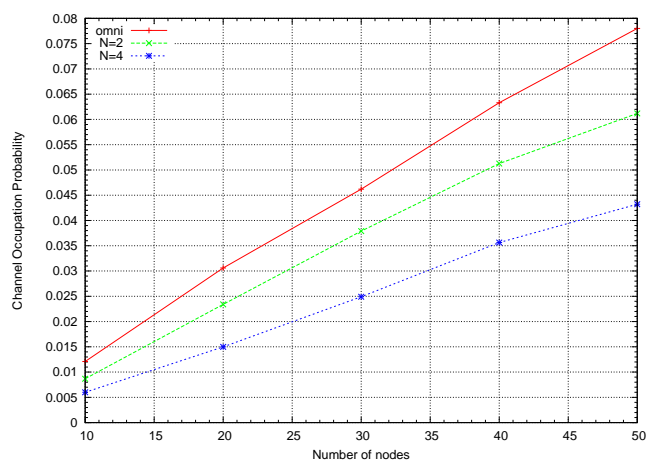

Fig. 6. Channel occupation probability as a function of the number of nodes in the case of omnidirectional and directive antennas with $\pi$ and $\pi / 2$ mainlobes for $T_{f}=93 \mathrm{~s}$ and $\delta=3 \%$. 